\title{
A prospective study comparing two embryo-transfer soft catheters
}

\author{
Emily De Conto ${ }^{1}$, Artur K Schuster ${ }^{1}$, Vanessa K Genro ${ }^{1}$, Rita Chapon ${ }^{1}$, Daniela S da Silva ${ }^{2}$, João Sabino Cunha- \\ Filho $^{2}$ \\ ${ }^{1}$ Federal University of Rio Grande do Sul - UFRGS - Porto Alegre - Brazil \\ 2 INSEMINE Center of Human Reproduction, Porto Alegre/RS - Brazil
}

\begin{abstract}
Objectives: To compare reproductive outcomes using two different soft catheters i.e. Set TDT $^{\circledR}$ and Cook $^{\circledR}$ Sydney IVF. The primary outcome was defined as a positive B-human chorionic gonadotropin (B-hCG) test.

Methods: Our prospective study recruited 68 patients undergoing in vitro fertilization cycles in a private fertility clinic in Porto Alegre, Brazil, between January 2014 and April 2016. They were divided into two groups according to the catheter that would be used for the embryo transfer, and the groups were matched by age. The total number of patients in each group was: 34 for the TDT and 34 for the Cook Sydney. All the patients were submitted to a B-hCG test 12 days after the embryo transfer for pregnancy outcome evaluation.

Results: Ten out of 34 patients from the TDT group had a positive outcome for pregnancy, corresponding to $29.4 \%$. The Cook Sydney group had 9 patients out of 34 with positive outcomes, corresponding to $26.5 \%$. Comparing the efficacy of both catheters for the primary outcome, there was no significant difference $(p>0.05)$ between the TDT and the Cook Sydney catheters.

Conclusion: The TDT and the Cook Sydney catheters efficacies were similar for embryo transfer during assisted reproductive technology cycles.
\end{abstract}

Keywords: Catheters, embryo transfer, pregnancy rate, assisted reproduction technology

\section{INTRODUCTION}

Embryo transfer (ET) is the last stage, and one of the most critical steps in Assisted Reproduction Technology (ART) cycles (Sallam, 2005). Among the most important variables that determine the success or failure of the procedure are: ultrasound guidance (Brown et al., 2016), uterine contractions during ET (Pierzyński \& ZbuckaKretowska, 2014), and the catheter type used during the procedure (Buckett, 2006). A few years ago, the type of catheter chosen was recognized as one of the main variables interfering positively or negatively on the ET effectiveness. Kovacs (1999) and Salha et al. (2001) classify the selection of the catheter, respectively, and third and fourth most important variables for the positive outcome of the cycle.

Considering the importance of the catheter chosen, some studies have been carried out to compare the efficacy of those commercially available. The results of these studies showed that the soft catheters had better performance when compared to rigid or semi-rigid ones (Abou-Setta et al., 2005; Ruhlmann et al., 2015), because they prevented trauma to the endocervix or the endometrium, and were pliable to be freely driven into the uterine cavity (Omidi et al., 2015). When soft catheters were compared, the most used, and therefore the most studied, were the following: Edwards-Wallace (McIlveen et al., 2005; Saldeen et al., 2008; Rhodes et al., 2007), Cook K-Jet (McIlveen et al., 2005), Frydman Ultrasoft (Ruhlmann et al., 2015) and Cook Sydney (Saldeen et al., 2008).

The preferred catheter varies according to each professional's experience in embryo transfer. In our fertility clinic, two types of soft catheters are the most used: the Tight Difficult Transfer Set (Set TDT ${ }^{\circledR}$ ) 4.5 and the Cook ${ }^{\circledR}$ Sydney IVF catheters. We have used these catheters in most ET procedures and found that there were no studies comparing their effectiveness; thus, we decided to run a prospective study to compare two groups of patients undergoing embryo transfers using the TDT 4.5 and the Cook ${ }^{\circledR}$ Sydney catheters.

\section{MATERIALS AND METHODS}

\section{Patients}

Sixty-eight women undergoing in vitro fertilization (IVF) cycles in our private fertility clinic (INSEMINE) in Porto Alegre, Brazil, were recruited for this prospective study. The recruitment period occurred between January 2014 and April 2016. The following criteria were considered for patient inclusion: regular ovulatory menstrual cycles every 25-35 days; both ovaries present; no clinical signs of hyperandrogenism; no current or past disease affecting ovaries or gonadotropin production and release, or sex steroid secretion, clearance or excretion; no current hormone therapy. Exclusion criteria involved: infertility caused by severe male factor and interrupted ART cycles. The age of the patients varied from 30 to 39 years. The most common cause of infertility among the patients was the male factor $(38.2 \%, n=26)$, followed by endometriosis $(37.9 \%, n=19)$, tubal factor $(19.1 \%, n=13)$ and other causes $(14.8 \%, \mathrm{n}=10)$.

The patients were divided into two groups according to the catheter that would be used for their ET: Set TDT (group 1) and Cook Sydney IVT (group 2). The choice of catheter was made based on the operator's personal experience. Each group had a total of 34 patients.

This study was analyzed and approved by our institutional review board. All the patients signed consent forms and were made aware that their data could be used in trials that intended to improve AR.

Hormonal Measurements and Laboratory Results The primary outcome was defined as a positive B-human chorionic gonadotrophin ( $B-h C G$ ) blood test. All the patients were submitted to this test 12 days after the ET. We considered as positive all the tests that had shown results above $25 \mathrm{mIU} / \mathrm{mL}$. We also collected the following data: age, cause of infertility, antral follicular count (AFC), follicle-stimulating hormone (FSH), antiMüllerian hormone (AMH), gonadotropin initial dose, number of follicles with diameter $\geq 17 \mathrm{~mm}$, retrieved oocytes, oocytes at metaphase II, fertilized oocytes in 
Table 1. Comparison between two catheters for embryo transfer in relation to patient data and IVF cycle outcomes

\begin{tabular}{|l|c|c|c|}
\hline \multirow{2}{*}{} & Set TDT & Catheters & \multirow{2}{*}{$\boldsymbol{p}$ value } \\
\cline { 2 - 4 } & 34 & 34 & - \\
\hline Number of AR cycles (n) & $34.0 \pm 2.9$ & $34.0 \pm 2.9$ & 1.000 \\
\hline AFC $(y)$ & $11.93 \pm 5.1$ & $11.82 \pm 6.4$ & 0.244 \\
\hline FSH (IU/L) & $7.33 \pm 2.28$ & $9.35 \pm 3.49$ & 0.068 \\
\hline AMH (pmol/L) & $3.99 \pm 3.54$ & $4.15 \pm 4.05$ & 0.921 \\
\hline Gonadotropin initial dose (IU) & $258.6 \pm 51.4$ & $234.4 \pm 67.5$ & 0.040 \\
\hline Follicles with diameter $\geq 17 \mathrm{~mm}$ & $4.88 \pm 3.7$ & $5.19 \pm 3.5$ & 0.988 \\
\hline Oocytes retrieved & $8.00 \pm 4.6$ & $7.69 \pm 3.8$ & 0.199 \\
\hline Number at metaphase II & $6.85 \pm 3.6$ & $6.38 \pm 3.3$ & 0.436 \\
\hline Fertilized oocytes in 2 PN & $3.56 \pm 2.8$ & $3.76 \pm 2.3$ & 0.159 \\
\hline Generated embryos & $4.21 \pm 2.7$ & $4.88 \pm 2.9$ & 0.823 \\
\hline Mean of embryo score & $66.9 \pm 24.3$ & $66.1 \pm 24.1$ & 0.773 \\
\hline Number of embryos transferred & $1.79 \pm 0.48$ & $1.97 \pm 0.31$ & 0.078 \\
\hline Pregnancy rate (\%) & 29.4 & 26.5 & 1.000 \\
\hline
\end{tabular}

The results are presented as mean \pm standard deviation. AFC: Antral Follicular Count, FSH: Follicle Stimulating Hormone, AMH: anti-Mullerian Hormone.

$2 \mathrm{PN}$, embryos generated, embryo score and number of embryos transferred (Table I).

Each woman was submitted to blood sampling procedure by venipuncture on cycle day 3 . The antagonist protocol was used for ovulation induction. We determined serum FSH levels using an automated multi-analysis system with chemiluminescence detection. Serum AMH levels were measured by an ultrasensitive enzyme-linked immunosorbent assay (ELISA). Laboratory data was obtained after oocyte puncture and embryo evaluation was performed according to Fisch et al. (2001) criteria.

\section{Catheters}

Set TDT $^{\circledR}$ (Laboratoire CCD, France) is an extra thin soft transfer catheter. It has an outer sheath with a flexible extremity and 2 hysterometry guide-marks at $5.5 \mathrm{~cm}$ and $6.5 \mathrm{~cm}$ from the distal end. It also has a malleable metal stylet coated in polyethylene and an extra-thin transfer catheter on a stainless steel micro tube of $0.5 \mathrm{~mm}$ in diameter in its proximal section.

The Cook $^{\circledR}$ Sydney IVF (Cook Medical, USA) has an outer firm portion and an inner ultra soft portion. The outer catheter is $19 \mathrm{~cm}$ long and has a polycarbonate hub, a bulb tip and an angled distal end. The inner portion is $23 \mathrm{~cm}$ long and the tip is 2.8 French size.

\section{Embryo transfers}

Embryo transfers were performed by a team of professionals formed by three gynecologists, all with over 5 years of experience. The difficult embryo transfers were excluded because they could influence the assisted reproduction cycle results.

\section{Statistical Analysis}

We used the Statistical Package for Social Sciences (SPSS Inc., Chicago, IL, USA) Statistics 20.0 software for statistical analysis purposes. The statistical analysis for categorical variables was made using the Pearson ChiSquared test. Continuous variables were analyzed by the
Student's $t$ test. The value considered as statistically significant was $p \leq .05$.

\section{RESULTS}

The study groups were matched by age and the following variables: AFC, FSH, AMH, number of follicles with diameter $\geq 17 \mathrm{~mm}$, retrieved oocytes, oocytes in metaphase II, oocytes in 2 PN, generated embryos, mean score of embryos and number of embryos transferred were not statistically significant. Ten out of thirty-four patients in the TDT group had a positive outcome for pregnancy, corresponding to $29.4 \%$. The Cook Sydney group had 9 patients out of 34 with positive outcomes, corresponding to $26.5 \%$. Comparing the efficacy of both catheters for the primary outcome, there was no significant difference $(p>0.05)$ between the TDT and the Cook Sydney catheters. The initial dose of gonadotropin reflects the ovarian reserve, and it was the only variable that showed statistical significance $(p=0.040)$, although all patients were subjected to the same treatment regimen. The final dosage was not significant $(p>0.05)$ in both groups. The primary outcome showed no difference between the groups. The statistical analysis results are depicted on Table I.

\section{DISCUSSION}

The superiority of flexible catheters over rigid and semi-rigid ones is well established in the literature. AbouSetta et al. (2005) in their review, concluded that flexible catheters have better pregnancy rates when compared to rigid catheters, and Ruhlmann et al. (2015) demonstrated that the implantation rate is increased when using flexible catheters rather than semi-rigid ones.

Several studies compared soft catheters. When the Cook Soft and the Edwards-Wallace catheters were compared, there was no statistically significant difference between them regarding pregnancy rates (Boone et al., 2001). Another study comparing those two flexible 
catheters was carried out by McIlveen et al. (2005), with a larger sample (75 patients per group) and they also found no significant difference in pregnancy rates between the groups. Rhodes et al. in 2007, comparing the same two types of catheters, showed that pregnancy rates with the Cook's was 5\% higher than with the Wallace's: $63.3 \%$ vs. $58.0 \%$, respectively; but the study power was not high enough to demonstrate statistically significant difference. van Weering et al. (2002) studied pregnancy rates comparing the TDT embryo transfer set and the Cook K-soft 5000 soft trans universal embryo transfer set, and found that pregnancy rates were significantly higher in the second group.

According to a literature review we carried out, the studies that compared the Cook and the Wallace soft catheters showed no statistical difference in efficacy between them (Boone et al., 2001; McIlveen et al., 2005; Rhodes et al., 2007; Saldeen et al., 2008). Just one study reported on the TDT Set (Buckett, 2006) and we did not find studies that compared the two types of catheters we compared.

Our study compared two types of soft embryo transfer catheters, with pregnancy rates of $29.4 \%$ and $26.5 \%$, for the TDT Set and the Cook Sydney, respectively. We found no significant difference between the groups $(p>0.05)$. The groups were matched by age, they used the same ovarian stimulation protocol and underwent the same laboratory procedures. Although there was a statistically different initial dose of gonadotropin between the groups, it did not impact our primary outcome (pregnancy rate): subsequent laboratory data related to ovulation induction was similar for both groups. Thus, we had a homogeneous sample in hormonal and laboratory terms, which allowed us to assess whether there was a correlation between the catheter used for embryo transfer and the B-hCG outcome. We believe that the main weaknesses of this study were the sample size and the fact that it was not a randomized clinical trial (RCT).

\section{CONCLUSION}

In conclusion, this prospective study supported that the modern ET soft catheters TDT and Cook Sydney Set had a similar performance in ART procedures. Catheter choice may be based on other factors, rather than its performance on outcomes. Future RCT studies concerning catheter types and their efficacy under ultrasound guided transfer are warranted.

\section{CONFLICT OF INTERESTS}

The authors declare that there was no potential conflict of interests, of financial or other nature, which may be deemed to influence the objectivity of this paper.

\section{Corresponding author:}

Emily De Conto

Hospital das Clínicas de Porto Alegre - HCPA/Universidade Federal do Rio Grande do Sul - UFRGS Porto Alegre, RS, Brazil.

E-mail: emydconto@yahoo.com.br

\section{REFERENCES}

Abou-Setta AM, Al-Inany HG, Mansour RT, Serour GI, Aboulghar MA. Soft versus firm embryo transfer catheters for assisted reproduction: a systematic review and metaanalysis. Hum Reprod. 2005;20:3114-21. PMID: 16040620 DOI: $10.1093 /$ humrep/dei198

Boone WR, Johnson JE, Blackhurst DM, Crane MM 4th. Cook versus Edwards-Wallace: are there differences in flexible catheters? J Assist Reprod Genet. 2001;18:15-7. PMID: PMC3455811 DOI: 10.1023/A:1026490327506
Brown J, Buckingham K, Buckett W, Abou-Setta AM. Ultrasound versus 'clinical touch' for catheter guidance during embryo transfer in women. Cochrane Database Syst Rev. 2016;3:CD006107. PMID: 26984325 DOI: 10.1002/14651858.CD006107.pub4

Buckett WM. A review and meta-analysis of prospective trials comparing different catheters used for embryo transfer. Fertil Steril. 2006;85:728-34. PMID: 16500345 DOI: $10.1016 /$ j.fertnstert.2005.08.031

Fisch JD, Rodriguez H, Ross R, Overby G, Sher G. The Graduated Embryo Score (GES) predicts blastocyst formation and pregnancy rate from cleavage-stage embryos. Hum Reprod. 2001;16:1970-5. PMID: 11527907

Kovacs GT. What factors are important for successful embryo transfer after in-vitro fertilization? Hum Reprod. 1999;14:590-2. PMID: 10221680 DOI: https://doi.org/10.1093/humrep/14.3.590

McIlveen M, Lok FD, Pritchard J, Lashen H. Modern embryo transfer catheters and pregnancy outcome: a prospective randomized trial. Fertil Steril. 2005;84:996-1000. PMID: 16213855 DOI: $10.1016 /$ j.fertnstert.2005.06.018

Omidi M, Halvaei I, Mangoli E, Khalili MA, Razi MH. The effect of embryo catheter loading technique on the live birth rate. Clin Exp Reprod Med. 2015;42:175-80. PMID: 26815646 DOI: 10.5653/cerm.2015.42.4.175

Pierzyński $\mathrm{P}$, Zbucka-Kretowska M. Uterine contractile activity at embryo transfer-as a new pharmacotherapeutic target in assisted reproduction. Ginekol Pol. 2014;85:60913. PMID: 25219142 DOI: $10.17772 / \mathrm{gp} / 1780$

Rhodes TL, Higdon HL 3rd, Boone WR. Comparison of pregnancy rates for two embryo-transfer catheters. Fertil Steril. 2007;87:411-6. PMID: 17094973 DOI: $10.1016 /$ j.fertnstert.2006.06.030

Ruhlmann C, Gnocchi DC, Cattaneo AR, Molina LG, Rivadeneira LR, Tessari L, Martínez AG. Embryo Transfer Catheters: SofterisEasier. JBRAAssistReprod. 2015;19:2049. PMID: 27203193 DOI: 10.5935/1518-0557.20150040

Saldeen P, Abou-Setta AM, Bergh T, Sundström P, Holte J. A prospective randomized controlled trial comparing two embryo transfer catheters in an ART program. Fertil Steril. 2008;90:599-603. PMID: 18054000 DOI: $10.1016 /$ j.fertnstert.2007.06.085

Salha OH, Lamb VK, Balen AH. A postal survey of embryo transfer practice in the UK. Hum Reprod. 2001;16:686-90. PMID: 11278219 DOI: https://doi.org/10.1093/humrep/16.4.686

Sallam HN. Embryo transfer: factors involved in optimizing the success. Curr Opin Obstet Gynecol. 2005;17:289-98. PMID: 15870564

van Weering $H G$, Schats $R$, McDonnell J, Vink JM, Vermeiden JP, Hompes PG. The impact of the embryo transfer catheter on the pregnancy rate in IVF. Hum Reprod. 2002;17:666-70. PMID: 11870120 DOI: https://doi.org/10.1093/humrep/17.3.666 